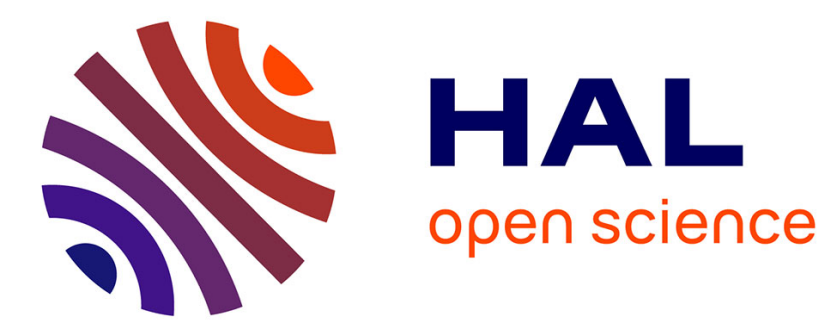

\title{
Stubborn state observers for linear time-invariant systems
}

\author{
Angelo Alessandri, Luca Zaccarian
}

\section{To cite this version:}

Angelo Alessandri, Luca Zaccarian. Stubborn state observers for linear time-invariant systems. Automatica, 2018, 88, pp.1 - 9. 10.1016/j.automatica.2017.10.022 . hal-01700167

\section{HAL Id: hal-01700167 \\ https://hal.laas.fr/hal-01700167}

Submitted on 6 Feb 2018

HAL is a multi-disciplinary open access archive for the deposit and dissemination of scientific research documents, whether they are published or not. The documents may come from teaching and research institutions in France or abroad, or from public or private research centers.
L'archive ouverte pluridisciplinaire HAL, est destinée au dépôt et à la diffusion de documents scientifiques de niveau recherche, publiés ou non, émanant des établissements d'enseignement et de recherche français ou étrangers, des laboratoires publics ou privés. 


\title{
Stubborn State Observers for Linear Time-invariant Systems
}

\author{
Angelo Alessandri ${ }^{\mathrm{a}}$, Luca Zaccarian ${ }^{\mathrm{b}}$ \\ ${ }^{a}$ Dipartimento di Ingegneria Meccanica, University of Genoa, P.le Kennedy Pad. D, I-16129 Genova, Italy \\ ${ }^{\mathrm{b}} L A A S-C N R S$, Université de Toulouse, CNRS, Toulouse, France, \\ and Dipartimento di Ingegneria Industriale, University of Trento, Italy
}

\begin{abstract}
For the purpose of estimating the state of a linear time-invariant system with measurements subject to outliers, we propose an observer with a saturated output injection in such a way to mitigate the effect of abnormal and isolated measurement noise on the error dynamics. Stability conditions in both the continuous-time and the discrete-time cases are derived, which ensure global exponential stability to the origin for the error dynamics. Such conditions can be expressed in terms of linear matrix inequalities, allowing for a viable design by using convex optimization. The effectiveness of the approach is illustrated by means of simulations in comparison with the Luenberger observer.
\end{abstract}

Key words: State observer, saturation, Outlier

\section{Introduction}

The long-standing problem of estimating the state variable of a plant was brilliantly solved in 1964 by Luenberger, who proposed in [19] to use a dynamic setup, called observer, which provides asymptotic state estimates under the action of an output injection (see also [18] for a further development). This pioneering result was extended from linear time-invariant (LTI) continuous-time systems to nonlinear and/or discretetime systems with a huge, still growing literature. The observers for LTI systems have a linear structure, which is well-suited for verifying the asymptotic stability of the estimation error. In this paper, we focus on observers for continuous-time and discrete-time LTI plants with a nonlinear output injection because of a saturation that depends on a variable threshold. The reason why we propose this novel estimation paradigm is that we aim at reducing the effect of measurement outliers, i.e., impulsive disturbances that may irremediably corrupt the measurements used for the purpose of state estimation.

\footnotetext{
* The material in this paper was partially presented at the 2015 European Control Conference, July 15-17, 2015, Linz, Austria.

${ }^{\star \star}$ Work supported in part by grant PowerLyap funded by the CaRiTRo.

Email addresses: alessandri@dime.unige.it (Angelo Alessandri), zaccarian@laas.fr (Luca Zaccarian).
}

The literature on estimation in the presence of outliers is vast but it refers mainly to identification problems. Most of the results rely on the idea of setting the Kalman filter so as to make it robust to outliers (see, among others, $[9,22,8])$. In [1] statistical tests are proposed that are less sensitive to abnormal noises. Identification based on an $l_{1}$ criterion is addressed in $[16,27]$. Instead of only attenuating the effect of outliers, a different approach is reported in [2], where a method based on a leave-one-out moving-horizon estimation strategy is proposed.

In the past two decades suitable characterizations of input saturation in control systems have been developed, allowing to reach out beyond the mere application of absolute stability concepts and global sector properties of the saturation nonlinearity [15]. In particular, according to the results in [23], it was recognized that global exponential stabilization of a linear plant using a bounded input is impossible, unless the plant is already exponentially stable. Therefore, suitable generalizations of the standard (globally-based) absolute stability results were proposed in $[13,10,14]$, which allowed to establish local results with a guaranteed region of attraction by way of a generalized (or local/regional) sector condition satisfied by the saturation nonlinearity (see also [24,12]). This generalized sector condition is nowadays a well known tool to address analysis and design of control laws for linear systems subject to saturations and deadzones.

In line with [3], here we employ a saturation function 
in the output injection signal of a linear asymptotic observer for a linear plant. As compared to the typical use of saturations, the novelty consists in using the saturation level artificially and adjusting it in such a way that global asymptotic stability properties of the error dynamics are guaranteed for any detectable linear plant. The adaptive saturation of the output error makes it possible to deal with specific types of measurement disturbances, such as the ones characterized by rare outliers. We call the resulting observers stubborn because indeed the saturation threshold is guaranteed to converge to zero in the absence of noise, and then possible outliers do not directly reach the error dynamics because they are mitigated by the limiting effect of saturation. On the other hand, persistent estimation errors gradually cause an increase of the saturation threshold and become increasingly important in the error dynamics, so as to guarantee global asymptotic stability of the origin for the estimation error dynamics. Interestingly, for the continuous-time case we can strengthen the global asymptotic stability (GAS) results to global exponential stability (GES) of the origin. This is however not the case for the parallel discrete-time results, where a significantly different proof technique must be adopted and the weaker GAS property of the origin can be established. We provide convex conditions for the selection of the observer parameters that can be expressed by means of linear matrix inequalities (LMIs) and easily solved by using semidefinite programming (SDP) tools [5]. Moreover, we prove that such conditions are always feasible under the (necessary) assumption that the plant state is detectable from the available measurement output. Finally, it is worth mentioning that there exist some few results on saturated high-gain observers for nonlinear systems. In [17], the saturation is used to ensure that the estimate takes into account the knowledge on the boundedness of the state trajectories. A nested-saturation lowpower approach is proposed in $[4,25]$ to overcome the well-known issue of the peaking, which affects high-gain observers.

Preliminary results of this work were presented in [3] only for the continuous-time case with a first proposal of tuning procedure for the observer parameters. Here, in addition to extending the results to the discrete-time case, we provide the proofs that were missing in [3], we discuss a novel tuning procedure for the observer parameters, and we illustrate the effectiveness of the proposed strategy on new simulation studies. We note that the discrete-time case discussed here presents somewhat different challenges for the proof of stability, which must follow a different paradigm.

The paper is structured as follows. The main results concerning the proposed stubborn estimator in the continuous-time and discrete-time cases are presented in Sections 2 and 3, respectively. Since numerical results in the first case are reported in [3], novel simulation results concerning only the second case for discrete- time LTI systems are shown in Section 4. Based on the foregoing, conclusions are drawn in Section 5.

Notation. The minimum and maximum eigenvalues of a real, symmetric matrix $P$ are denoted by $\lambda_{\min }(P)$ and $\lambda_{\max }(P)$, respectively; in addition, $P>0, P \geq 0, P<0, P \leq 0$, means that $P$ is positive definite, positive semidefinite, negative definite, and negative semidefinite, respectively. Given a generic matrix $M, \operatorname{He} M:=M^{\top}+M$ and $|M|:=\left(\lambda_{\max }\left(M^{\top} M\right)\right)^{1 / 2}=\left(\lambda_{\max }\left(M M^{\top}\right)\right)^{1 / 2}$. Finally, let $(x, y):=\left[x^{\top}, y^{\top}\right]^{\top}$, where $x$ and $y$ are two vectors.

\section{Main results for continuous-time LTI systems}

\subsection{Observer architecture}

We consider an LTI continuous-time plant with state $x \in \mathbb{R}^{n}$, output $y \in \mathbb{R}^{n_{y}}$, control input $u \in \mathbb{R}^{n_{u}}$, and disturbance $d \in \mathbb{R}^{n_{d}}$ :

$$
\begin{aligned}
& \dot{x}=A x+B_{u} u+B_{d} d \\
& y=C x+D_{u} u+D_{d} d .
\end{aligned}
$$

In this paper, we propose the following dynamic state estimator:

$$
\begin{aligned}
& \dot{\hat{x}}=A \hat{x}+B_{u} u+L \operatorname{sat}_{\sigma}(y-\hat{y}) \\
& \hat{y}=C \hat{x}+D_{u} u,
\end{aligned}
$$

where sat $_{\sigma}$ is a symmetric vector saturation function with variable non-negative saturation limits $\sigma=\left(\sigma_{1}, \cdots, \sigma_{n_{y}}\right) \in \mathbb{R}_{\geq 0}^{n_{y}}$ defined for each $s=$ $\left(s_{1}, \cdots, s_{n_{y}}\right) \in \mathbb{R}^{n_{y}}$ as

$\operatorname{sat}_{\sigma}(s)=\left[\begin{array}{c}\operatorname{sat}_{\sigma_{1}}\left(s_{1}\right) \\ \vdots \\ \operatorname{sat}_{\sigma_{n_{y}}}\left(s_{n_{y}}\right)\end{array}\right]$,

$\operatorname{sat}_{\sigma_{k}}\left(s_{k}\right)=\max \left\{-\sigma_{k}, \min \left\{\sigma_{k}, s_{k}\right\}\right\}$ being the standard scalar symmetric saturation function. The observer dynamics is completed by the following scalar equation governing the evolution of the saturation limits $\sigma$ :

$$
\begin{aligned}
\dot{\bar{\sigma}} & =-\lambda \bar{\sigma}+(y-\hat{y})^{\top} R(y-\hat{y}), \quad \bar{\sigma} \in \mathbb{R}_{\geq 0} \\
\sigma_{i} & =\sqrt{\bar{\sigma} / w_{i}}, \quad i=1, \ldots, n_{y},
\end{aligned}
$$

where $\lambda>0$ scalar, $R=R^{\top}>0$ and $w_{i}>0, i=$ $1, \ldots, n_{y}$ are suitable parameters. Moreover, by $\bar{\sigma} \in \mathbb{R}_{\geq 0}$ we mean that the dynamics of $\bar{\sigma}$ is constrained to the 
non-negative real axis, which is indeed forward invariant for dynamics (2c). This constraint clearly makes the square root in $(2 \mathrm{~d})$ well posed.

The rationale behind the nonlinear observer (2) is that of a "stubborn" observer, in the sense that the output estimation error $e_{y}:=y-\hat{y} \in \mathbb{R}^{n_{y}}$ is injected in the observer equations (2a) through a bounded function. The advantage of such an observer structure is that possible outliers in the measurements are suitably canceled (actually saturated) and their effect on the estimation error is reduced. Dynamic adaptation of the saturation level is then necessary in such a way that global asymptotic stability of the estimation error dynamics can be ensured. In particular, in the adaptation equation $(2 \mathrm{c})$, the effect of the first term (involving $\lambda$ ) is to push to zero the saturation levels $\sigma$, while the effect of the second term (depending on $R$ ) is to ensure that an output estimation error eventually leads to a suitable increase of $\sigma$ in such a way that the error dynamics can be stabilized.

\subsection{Stability and feasibility}

Given the observer architecture (2), we provide here necessary conditions on the observer parameters $L, \lambda, R$, and $W$ that ensure uniform global asymptotic stability of the dynamics of the error variables $(e, \bar{\sigma})$, where $e:=x-\hat{x}$, in the absence of disturbances, i.e., with $d=0$. This dynamics can be easily computed from (1) and (2) as follows

$$
\begin{aligned}
\dot{e} & =(A-L C) e+L q \\
\dot{\bar{\sigma}} & =-\lambda \bar{\sigma}+e^{\top} C^{\top} R C e, \quad \bar{\sigma} \in \mathbb{R}_{\geq 0} \\
q & =\mathrm{dz}_{\sigma}(C e):=C e-\operatorname{sat}_{\sigma}(C e),
\end{aligned}
$$

where the deadzone function $\operatorname{dz}_{\sigma}(s):=s-\operatorname{sat}_{\sigma}(s)$ and the auxiliary variable $q \in \mathbb{R}^{n_{y}}$ are introduced to simplify the notation.

Remark 1 Note that the adaptation law in (2c) is necessary if one wants to propose an observer with a saturated output injection for general linear plants. Indeed, the simple alternative of selecting a constant (or bounded) saturation level $\sigma$ would dramatically fail to work whenever the matrix A has eigenvalues with positive real part. This is apparent if one realizes that the error dynamics (3) corresponds to a linear plant with a saturated linear output injection and, as established in [23], exponentially unstable linear dynamics cannot be globally asymptotically stabilized through a bounded input. In this paper we propose the dynamic saturation level in (2c), (2d) which instead makes our solution applicable to any linear plant, as long as the pair $(C, A)$ is detectable, as established later in Proposition 1.

Below, we state our main result, where the observer parameters will be designed according to a few convex con- straints. A first constraint ensures that the interplay between parameters $\lambda$ and $R$ of the adaptation forces $\bar{\sigma}$ to grow fast enough in such a way as to prevent the divergence of a suitable quadratic Lyapunov function $e^{\top} P e$ :

$$
\mathrm{He}\left[\begin{array}{cc}
P A-X C+\frac{1}{2}\left(\lambda P-C^{\top} R C\right) & X \\
U C & -U
\end{array}\right]<0 .
$$

with $P=P^{\top}>0 X \in \mathbb{R}^{n \times n_{y}}$ is an auxiliary variable allowing us to linearize the dependence on the output injection gain $L$, and where $U>0$ is a diagonal scaling matrix introduced to have extra degrees of freedom for parameter optimization.

Two constraints will be imposed to ensure that, once $\bar{\sigma}$ has grown large enough to "capture" the error, both the error $e$ and the saturation level $\sigma$ will actually converge to zero:

$$
\begin{aligned}
& \text { He }\left[\begin{array}{cc}
P A-X C & X \\
W C+Y & -W
\end{array}\right]<0 \\
& {\left[\begin{array}{cc}
P & Y_{i}^{\top} \\
Y_{i} & w_{i}
\end{array}\right] \geq 0, \quad i=1, \ldots, n_{y},}
\end{aligned}
$$

where $w_{i}$ denotes the $i$-th diagonal entry of the diagonal positive definite matrix $W>0$ (i.e., $W$ := $\left.\operatorname{diag}\left(w_{1}, \ldots, w_{n_{y}}\right)\right)$ and $Y_{i}$ denotes the $i$-th row of matrix $Y \in \mathbb{R}^{n_{y} \times n}$. Our main stability result follows.

Theorem 1 Consider any feasible solution to the matrix inequalities (4) in the variables $X, Y, \lambda>0, P=P^{\top}>$ $0, R=R^{\top}>0, U>0$ diagonal, $W>0$ diagonal, and use them as parameters $R, W, \lambda$ for observer (2), together with

$$
L=P^{-1} X .
$$

Then the error dynamics (3) associated with the observerplant interconnection (1)-(2) is globally exponentially stable to the origin.

Remark 2 Note that Theorem 1 claims that GES (global exponential stability) holds in both state variables e and $\bar{\sigma}$, thereby establishing useful uniform boundedness and exponential convergence to zero for both the error e and the saturation levels $\sigma$ (see equation $(2 \mathrm{~d})$ ).

Proof. We prove the theorem by introducing a suitable non-differentiable Lipschitz Lyapunov function $V$. Following [26], we only need to establish a flow condition for almost all $x$ (namely, wherever the gradient of $V$ is defined). Indeed, around each point where the condition does not hold there is a full measure set of points where it holds. Then following the reasoning in [26, p. 100], we obtain that the Lyapunov condition holds for the generalized gradient [7] and asymptotic stability follows from 
the derivations in $[7,26]$. To define the Lyapunov function, first note that both inequalities (4a) and (4b) are strict so that one can find a small enough $\varepsilon>0$ such that

$$
\begin{array}{r}
\text { He } \left.\begin{array}{rc}
P A-X C+\frac{1}{2}\left(\lambda P-C^{\top} R C\right) & X \\
U C & -U
\end{array}\right]<-2 \varepsilon I, \\
\operatorname{He}\left[\begin{array}{cc}
P A-X C & X \\
W C+Y & -W
\end{array}\right]<-2 \varepsilon I .
\end{array}
$$

Then we define the Lyapunov function as

$$
V(e, \bar{\sigma})=\underbrace{e^{\top} P e+\zeta \bar{\sigma}}_{V_{1}(e, \bar{\sigma})}+\eta \underbrace{\max \left\{e^{\top} P e-\bar{\sigma}, 0\right\}}_{V_{2}(e, \bar{\sigma})}
$$

where $\zeta$ and $\eta$ are positive scalars selected below. Note that $V$ is positive definite in the set $\mathbb{R}^{n} \times \mathbb{R}_{>0}$ where the dynamics is constrained. Indeed, the first term is positive definite and the second term is non-negative. As mentioned above, the function $V$ is non-differentiable in the zero measure set where $e^{\top} P e-\bar{\sigma}=0$. Below, we prove the theorem by establishing the decrease of $V$ wherever it is differentiable. In particular, we split the analysis in two cases.

Case 1: $e^{\top} P e<\bar{\sigma}$. Here we will select the scalar $\zeta$ in (8). Define matrix $H=W^{-1} Y$. Then we can apply a Schur complement to (4c) and pre- and post-multiply by $e$ and its transpose to get $e^{\top} P e-w_{i} e^{\top} H_{i}^{\top} H_{i} e \geq 0$, which implies, together with the condition of Case 1 , and relation $(2 \mathrm{~d})$ :

$$
w_{i}\left|H_{i} e\right|^{2} \leq e^{\top} P e \leq \bar{\sigma}=w_{i} \sigma_{i}^{2}, \quad \forall i \in\left\{1, \ldots, n_{y}\right\}
$$

Dividing the inequality above by $w_{i}$ we obtain $\mathrm{dz}_{\sigma_{i}}\left(H_{i} e\right)=$ 0 for all $i \in\left\{1, \ldots, n_{y}\right\}$, which implies $\mathrm{dz}_{\sigma}(H e)=0$. Exploiting this property, we can use the following generalized (local) sector condition introduced in $[10,14]$ and reformulated in [24, Lemma 1.6] as follows:

$\mathrm{dz}_{\sigma}(H e)=0 \Rightarrow q^{\top} W(C e+H e-q) \geq 0$,

whose right hand inequality is therefore valid for all $(e, \bar{\sigma})$ satisfying the condition of Case 1.

Let us now use inequality (7), which can be pre- and post-multiplied by $(e, q)$ and its transpose, to get:

$2 e^{\top} P((A-L C) e+L q)+2 q^{\top} W(C e+H e-q)<-2 \varepsilon|e|^{2}$,

where we have used $X=P L$ (from (5)) and $Y=W H$ from the definition above. Finally, let us select

$$
\zeta=\frac{\varepsilon}{\left|C^{\top} R C\right|}
$$

and compute the variation of $V$ in (8) along the dynamics (3), which gives (note that the second term $V_{2}$ in (8) is identically zero in the set considered in Case 1 , so it gives no contribution to the equation below):

$$
\begin{aligned}
\dot{V}(e, \bar{\sigma}) & =2 e^{\top} P((A-L C) e+L q)+\zeta \dot{\bar{\sigma}} \\
& <-2 q^{\top} W(C e+H e-q)-2 \varepsilon|e|^{2}+\zeta \dot{\bar{\sigma}} \\
& \leq\left(-2 \varepsilon+\zeta\left|C^{\top} R C\right|\right)|e|^{2}-\lambda \zeta \bar{\sigma} \\
& \leq-\varepsilon|e|^{2}-\lambda \zeta \bar{\sigma}, \quad \text { if } e^{\top} P e<\bar{\sigma},
\end{aligned}
$$

where we have used (9) and (10).

Case 2: $e^{\top} P e>\bar{\sigma}$. Here we will select the scalar $\eta$ in (8). For the case considered here, the maximum term in the expression of $V_{2}$ in (8) is always the first one, therefore we may consider inequality (6), which can be multiplied on both sides by $(e, q)$ and its transpose, to obtain

$$
\begin{aligned}
\dot{V}_{2}(e, \bar{\sigma}) & =2 e^{\top} P((A-L C) e+L q)-e^{\top} C^{\top} R C e+\lambda \bar{\sigma} \\
& <-2 q^{\top} U(C e-q)-2 \varepsilon\left(|e|^{2}+|q|^{2}\right) \\
& \leq-2 \varepsilon\left(|e|^{2}+|q|^{2}\right),
\end{aligned}
$$

where in the first step we used $\bar{\sigma}<e^{\top} P e$ (from Case 2) and in the second step we used (9) with $H=0$, which clearly holds globally (because $\mathrm{dz}_{\sigma}(0)=0$ for any $\sigma$ ).

Consider now the upper left component of inequality (7), which implies $P(A-L C)<-2 \varepsilon I$, recall that (11) holds, and select

$$
\eta=\frac{|P L|}{2 \varepsilon}
$$

to get (some steps related to $\dot{\bar{\sigma}}$ being similar to those in $(12))$ :

$$
\begin{aligned}
\dot{V}(e, \bar{\sigma}) & =2 e^{\top} P(A-L C) e+2 e^{\top} P L q+\zeta \dot{\bar{\sigma}}+\eta \dot{V}_{2}(e, \bar{\sigma}) \\
& <\left(-2 \varepsilon+\zeta\left|C^{\top} R C\right|\right)|e|^{2}-\lambda \zeta \bar{\sigma}+2 e^{\top} P L q \\
& \leq-\varepsilon|e|^{2}-\lambda \zeta \bar{\sigma}+2|P L||e||q|-|P L|\left(|e|^{2}+|q|^{2}\right) \\
& \leq-\varepsilon|e|^{2}-\lambda \zeta \bar{\sigma}, \quad \text { if } e^{\top} P e>\bar{\sigma} .
\end{aligned}
$$

Summary. Based on the analysis in Cases 1 and 2 above and the resulting relations (12), and (14), we may prove GES by replacing $\bar{\sigma}$ with $\sigma$ as per (2d), which implies $|\sigma|^{2}=\bar{\sigma} \sum_{i=1}^{n_{y}} w_{i}^{-1}$, so that we may establish the following upper and lower bounds:

$$
\begin{aligned}
& c_{1}\left(|e|^{2}+|\sigma|^{2}\right) \leq V(e, \bar{\sigma}) \leq c_{2}\left(|e|^{2}+|\sigma|^{2}\right) \\
& c_{1}:=\min \left\{\lambda_{\min }(P), \zeta \frac{\lambda_{\min }(W)}{n_{y}}\right\} \\
& c_{2}:=\max \left\{(1+\eta) \lambda_{\max }(P), \zeta \frac{\lambda_{\max }(W)}{n_{y}}\right\} .
\end{aligned}
$$


Moreover, from (12) and (14) we obtain

$$
\dot{V}(e, \bar{\sigma}) \leq-c_{3}\left(|e|^{2}+|\sigma|^{2}\right), \text { for almost all } e, \sigma,
$$

where

$$
c_{3}=\min \left\{\varepsilon, \lambda \zeta \frac{\lambda_{\min }(W)}{n_{y}}\right\} .
$$

Inequalities above imply a uniform global exponential bound on $|e|^{2}+|\sigma|^{2}$, which can then be easily extended to $\bar{\sigma}$.

Remark 3 One property that is perhaps not evident from the proof of Theorem 1 is that solutions to (3) always reach in finite time the closed and unbounded set

$$
\mathcal{E}(P, \bar{\sigma}):=\left\{(e, \bar{\sigma}): e^{\top} P e \leq \bar{\sigma}\right\},
$$

which is forward invariant. Then they asymptotically converge to the origin. This fact can be established by following similar derivations to those in Case 2 of the proof of Theorem 1 while analyzing the function

$$
\psi_{c}(e, \bar{\sigma})=e^{\top} P e-(1-c) \bar{\sigma},
$$

with c selected as a sufficiently small positive scalar. Then it can be shown that there exists a sufficiently small $c$ such that $\psi_{c}(e, \bar{\sigma}) \geq 0$ implies $\dot{\psi}_{c}<0$, which means that solutions asymptotically converge to the set where $e^{\top} P e \leq(1-c) \bar{\sigma}$ and, as a consequence, they converge in finite time to the set (16). Moreover, forward invariance of (16) can be easily established by the properties of $\psi_{c}$ with $c=0$. It is useful to also emphasize that from this property it is not completely straightforward to prove $G A S$ of the origin, as the set $\mathcal{E}(P, \bar{\sigma})$ is unbounded, so that a suitable boundedness property of solutions must be proven to obtain GAS of the origin. This is indeed the proof technique adopted in the discrete-time version of our results, where the analysis cannot be elegantly split in two cases as in the proof of Theorem 1.

We state next a feasibility result showing that the proposed linear matrix inequality (LMI) conditions (4) always admit a feasible solution as long as pair $(C, A)$ is detectable (which is a necessary assumption for the existence of an asymptotic observer).

Proposition 1 Given plant (1), conditions (4) are feasible if and only if pair $(C, A)$ is detectable.

Proof. Necessity follows from standard detectability results. Indeed, if pair $(C, A)$ is not detectable, no asymptotic observer exists for the plant state and therefore from Theorem 1, conditions (4) must be infeasible.

To prove sufficiency, we first use [11, Theorem 16.6] to get that detectability implies the existence of $P=P^{\top}>0$ satisfying

$A^{\top} P+P A-C^{\top} C<0$.

Then we pre- and post-multiply (4a) and (4b) by $\left[\begin{array}{cc}I & C^{\top} \\ 0 & I\end{array}\right]$ and its transpose, respectively, to get the equivalent conditions:

Не $\left[\begin{array}{cc}P A-\frac{C^{\top} R C}{2}+\frac{\lambda}{2} P & X \\ -U C & -U\end{array}\right]<0$
He $\left[\begin{array}{cc}P A+C^{\top} Y & X \\ -W C+Y & -W\end{array}\right]<0$.

Now consider the selection

$$
Y=-\frac{C}{2}, U=W, R=I, X=C^{\top} W+\frac{C^{\top}}{2}
$$

and note that (18) and (4c) simplify to:

$$
\begin{aligned}
& {\left[\begin{array}{cc}
A^{\top} P+P A-C^{\top} C+\lambda P & C^{\top} / 2 \\
C / 2 & -2 W
\end{array}\right]<0} \\
& {\left[\begin{array}{cc}
A^{\top} P+P A-C^{\top} C & 0 \\
0 & -2 W
\end{array}\right]<0} \\
& {\left[\begin{array}{cc}
P & C_{i}^{\top} / 2 \\
C_{i} / 2 & w_{i}
\end{array}\right] \geq 0, \quad i=1, \ldots, n_{y},}
\end{aligned}
$$

which are clearly satisfied if one selects $P$ satisfying (17), $\lambda$ sufficiently small, and $W$ sufficiently large.

Remark 4 Theorem 1 ensures that the observer state $\bar{\sigma}$ converges exponentially to the origin. In the presence of noise and modeling uncertainty, this property does not hold anymore and one expects $\bar{\sigma}$ to not converge to zero, or even diverge. This property may be exploited in mode estimation for switching systems, where a pool of observers, one for each mode, is put in place [6]. In practice, one may regard $\bar{\sigma}$ as a monitoring signal that provides a measure of how good the estimator tracks the state of the system and possibly acts on the system by tuning the control.

The design of the proposed observer can be done by exploiting the effectiveness of popular convex optimization tools for LMIs. Some naive ideas are reported in [3]. The reader is referred to Section 3.3 in the next section for an overview on the possible approaches to construct stubborn observers since they can be adopted in both the continuous-time and the discrete-time cases. 


\section{Main results for discrete-time LTI systems}

\subsection{Observer architecture}

In this section, we follow parallel discrete-time derivations to those of Section 2.1. For describing discrete-time dynamics we simplify notation using the push-forward operator $x^{+}:=x(k+1)$ that allows omitting time " $k$ " from our equations (parallel to notation $\dot{x}:=\frac{d x(t)}{d t}$ ), thereby simplifying the following derivations. We start from the discrete-time plant

$$
\begin{aligned}
x^{+} & =A x+B_{u} u+B_{d} d \\
y & =C x+D_{u} u+D_{d} d,
\end{aligned}
$$

and use the observer

$$
\begin{aligned}
\hat{x}^{+} & =A \hat{x}+B_{u} u+L \operatorname{sat}_{\sigma}(y-\hat{y}) \\
\bar{\sigma}^{+} & =\lambda \bar{\sigma}+(y-\hat{y})^{\top} R(y-\hat{y}), \quad \bar{\sigma} \in \mathbb{R}_{\geq 0} \\
\hat{y} & =C \hat{x}+D_{u} u, \\
\sigma_{i} & =\sqrt{\bar{\sigma} / w_{i}}, \quad i=1, \ldots, n_{y},
\end{aligned}
$$

where $\lambda \in[0,1)$ scalar, $R=R^{\top}>0$ and $w_{i}>0$, $i=1, \ldots, n_{y}$ are suitable parameters. Once again we introduce $W:=\operatorname{diag}\left(w_{1}, \ldots, w_{n_{y}}\right)$.

\subsection{Stability}

For the discrete-time observer (21) we can study the stability of the estimation error $e=x-\hat{x}$, having dynamics whose right-hand side coincides with the corresponding continuous-time one in (3) under the absence of disturbances, i.e., with $d=0$ in (20). Such a dynamics of the estimation error is repeated here for simplified referencing:

$$
\begin{aligned}
e^{+} & =(A-L C) e+L q \\
\bar{\sigma}^{+} & =\lambda \bar{\sigma}+e^{\top} C^{\top} R C e, \quad \bar{\sigma} \in \mathbb{R}_{\geq 0},
\end{aligned}
$$

with $q$ defined as in (3).

We can now formulate parallel conditions to those in (4) to ensure GAS of the origin for the error dynamics (22). In particular, (4a) and (4b) transform into

$$
\operatorname{He}\left[\begin{array}{ccc}
-\frac{1}{2}\left(\lambda P+C^{\top} R C\right) & 0 & 0 \\
U C & -U & 0 \\
P A-X C & X & -\frac{1}{2} P
\end{array}\right]<0
$$

and

$$
\mathrm{He}\left[\begin{array}{ccc}
-\frac{1}{2} P & 0 & 0 \\
W C+Y & -W & 0 \\
P A-X C & X & -\frac{1}{2} P
\end{array}\right]<0
$$

respectively, while equation (4c) remains unchanged. The following theorem is the discrete-time version of Theorem 1.

Theorem 2 Consider any feasible solution to the matrix inequalities (4c), (23), and (24) in the variables $X, Y$, $\lambda \in[0,1), P=P^{\top}>0, R=R^{\top}>0, U>0$ diagonal, $W>0$ diagonal, and use them as parameters $R, W, \lambda$ for observer (21), together with the gain selection (5). Then the error dynamics (22) associated with the observerplant interconnection (20)-(21) is globally asymptotically stable to the origin.

Proof. The proof relies on discrete-time matrix manipulations parallel to the continuous-time ones adopted in the proof of Theorem 1. However, for the discrete-time case it is not possible to obtain such an elegant bound on the variation of the Lyapunov function $V$ in (8). Therefore, we resort to a different type of proof where we use the discrete-time version of the forward invariance property commented in Remark 3 and apply a suitable stability result for cascaded systems. More specifically, the proof is carried out in three steps:

- In step A) we show that the closed (but unbounded) set $\mathcal{M}:=\mathcal{E}(P, \bar{\sigma})$ defined in (16) is forward invariant and that the origin is globally asymptotically stable (GAS) for the dynamics restricted to this set.

- In step B) we show that the set $\mathcal{M}$ is stable and (not necessarily uniformly) attractive from the whole state space.

- In step C) we show that all solutions are bounded.

Applying the following corollary of [21, Lemma 1 and Remark 8], ${ }^{1}$ we then obtain GAS of the origin. The different proof technique and, in particular, the use of Lemma 1 has the consequence that for our discrete-time result we do not establish GES but only GAS of the origin. This is because the discrete-time bounds that we obtain do not lead to a single Lyapunov function with nice homogeneous bounds (as in Theorem 1). Whether the established GAS can be strengthened to GES remains an open question.

Lemma 1 [21] Consider a nonlinear system $\xi^{+}=f(\xi)$, where $f: \mathbb{R}^{n} \rightarrow \mathbb{R}^{n}$ is continuous, and assume that

(1) a closed set $\mathcal{M} \subset \mathbb{R}^{n}$ is stable and globally attractive (therefore forward invariant);

1 Note that [21, Lemma 1] is stated for continuous-time systems but [21, Remark 8] emphasizes that the related construction applies to hybrid systems satisfying certain hybrid basic conditions. Discrete-time systems with continuous right hand side fall into this family of hybrid systems and this is why Lemma 1 is a corollary of [21, Lemma 1 and Remark 8]. 
(2) the compact set $\mathcal{M}_{\circ} \subset \mathcal{M}$ is stable and globally attractive relative to $\mathcal{M}$ (namely, relative to initial conditions in $\mathcal{M}$ ).

Then the set $\mathcal{M} \circ$ is asymptotically stable for $\xi^{+}=f(\xi)$ with basin of attraction coinciding with the largest set of initial conditions from which all solutions are bounded. In particular, if all solutions are bounded, then the set $\mathcal{M}_{\circ}$ is globally asymptotically stable for $\xi^{+}=f(\xi)$.

Step (A). We first apply a Schur complement to (23) and (24), corresponding to inverting the $(3,3)$ block entry of both inequalities, and introduce strengthened versions of them, parallel to (6), (7), which hold for a sufficiently small $\varepsilon>0$. Below, we use relation $X=P L$ (from (5)) and we use the shortcut notation $A_{L}:=A-L C$ :

$$
\begin{gathered}
{\left[\begin{array}{cc}
A_{L}^{\top} P A_{L}-\lambda P-C^{\top} R C & \star \\
L^{\top} P A_{L}+U C & -2 U+L^{\top} P L
\end{array}\right]<-2 \varepsilon I,} \\
{\left[\begin{array}{cc}
A_{L}^{\top} P A_{L}-P & \star \\
L^{\top} P A_{L}+W C+Y & -2 W+L^{\top} P L
\end{array}\right]<-2 \varepsilon I,}
\end{gathered}
$$

where $\star$ denotes symmetric entries from now on. Then we consider the Lyapunov function $V$ in (8), used in the continuous-time case, and choose its parameter $\zeta$. Denoting again $H=W^{-1} Y$, we may apply a Schur complement to (4c) to obtain $e^{\top} P e-w_{i} e^{\top} H_{i}^{\top} H_{i} e \geq 0$ and following the same derivations as in the proof of Theorem 1 , we get $\mathrm{dz}(\mathrm{He})=0$, which implies that the local sector condition (9) holds.

Let us now use inequality (26), which can be pre- and post-multiplied by $(e, q)$ and its transpose, to get:

$$
\begin{aligned}
e^{\top}\left(A_{L}^{\top} P A_{L}-P\right) e+q^{\top} L^{\top} P\left(2 A_{L} e+L q\right) \\
+2 q^{\top} W(C e+H e-q)<-2 \varepsilon|e|^{2},
\end{aligned}
$$

where we used $Y=W H$ from the definition above. To show that the set $\mathcal{M}=\mathcal{E}(P, \bar{\sigma})$ in (16) is forward invariant, consider using $-\bar{\sigma} \leq-e^{\top} P e$ below, to get

$$
\begin{aligned}
& \left(e^{+}\right)^{\top} P e^{+}-\bar{\sigma}^{+} \\
& =\left(A_{L} e+L q\right)^{\top} P\left(A_{L} e+L q\right)-\left(\lambda \bar{\sigma}+e^{\top} C^{\top} R C e\right) \\
& \leq\left(A_{L} e+L q\right)^{\top} P\left(A_{L} e+L q\right)-e^{\top}\left(\lambda P+C^{\top} R C\right) e \\
& \quad+2 q^{\top} U(C e-q) \leq 0,
\end{aligned}
$$

where we used the global sector condition $2 q^{\top} U(C e-$ $q) \geq 0$ and where the last inequality is straightforward from hitting $(25)$ on both sides by $(e, q)$ and its transpose.
Finally, let us select

$$
\zeta=\frac{\varepsilon}{\left|C^{\top} R C\right|}
$$

and compute the variation of $V$ in (8) along dynamics (22). To this aim, we note that $V_{2}(e, \bar{\sigma})=0$ because we are restricting the dynamics to $\mathcal{M}$. and that also $V_{2}\left(e^{+}, \bar{\sigma}^{+}\right)=0$ due to the forward invariance of $\mathcal{M}$ established in (28). Then we obtain:

$$
\begin{aligned}
& \Delta V(e, \bar{\sigma}):=V_{1}\left(e^{+}, \bar{\sigma}^{+}\right)-V_{1}(e, \bar{\sigma})=e^{\top}\left(A_{L}^{\top} P A_{L}\right. \\
& -P) e+q^{\top} L^{\top} P\left(2 A_{L} e+L q\right)+\zeta\left(\bar{\sigma}^{+}-\bar{\sigma}\right) \\
& \quad<-2 q^{\top} W(C e+H e-q)-2 \varepsilon|e|^{2}+\zeta\left(\bar{\sigma}^{+}-\bar{\sigma}\right) \\
& \quad \leq\left(-2 \varepsilon+\zeta\left|C^{\top} R C\right|\right)|e|^{2}-(1-\lambda) \zeta \bar{\sigma} \\
& \quad \leq-\varepsilon|e|^{2}-(1-\lambda) \zeta \bar{\sigma}, \quad \text { if }(e, \bar{\sigma}) \in \mathcal{M},
\end{aligned}
$$

where we have used (27) and (9). This last inequality implies the negative definiteness of $\Delta V$ with respect to the origin for all states in $\mathcal{M}$, which implies GES, therefore also GAS, of the origin for the dynamics restricted to $\mathcal{M}$.

Step (B). To show that the set $\mathcal{M}$ is stable and globally attractive, we rely once again on function $V$ in (8), and choose its parameter $\eta$. Since we are studying stability properties of the forward invariant set $\mathcal{M}$, we are only interested in points of the state space where $\bar{\sigma}<e^{\top} P e$ (namely, points outside $\mathcal{M}$ ) and where $\bar{\sigma}^{+}<\left(e^{+}\right)^{\top} P e^{+}$ (namely, points mapped outside $\mathcal{M}$, indeed any point mapped inside $\mathcal{M}$ clearly leads to solutions converging to $\mathcal{M})$.

For this special case, the maximum term in the expression of $V_{2}$ in (8) is the first one both before and after the jump, therefore, by also using $\bar{\sigma} \leq e^{\top} P e$, we get

$$
\begin{aligned}
& \Delta V_{2}(e, \bar{\sigma})=\left(e^{+}\right)^{\top} P e^{+}-\bar{\sigma}^{+}-\left(e^{\top} P e-\bar{\sigma}\right) \\
& =e^{\top}\left(A_{L}^{\top} P A_{L}-P-C^{\top} R C\right) e+q^{\top} L^{\top} P\left(2 A_{L} e\right. \\
& \quad+L q)+(1-\lambda) \bar{\sigma} \\
& \leq e^{\top}\left(A_{L}^{\top} P A_{L}-\lambda P-C^{\top} R C\right) e+q^{\top} L^{\top} P\left(2 A_{L} e\right. \\
& \quad+L q) .
\end{aligned}
$$

The above equation can be combined with inequality (25) multiplied on both sides by $(e, q)$ and its transpose, to obtain

$$
\begin{aligned}
\Delta V_{2}(e, \bar{\sigma}) & \leq-2 q^{\top} U(C e-q)-2 \varepsilon\left(|e|^{2}+|q|^{2}\right) \\
& \leq-2 \varepsilon\left(|e|^{2}+|q|^{2}\right),
\end{aligned}
$$

where we have used (9) with $H=0$, which holds globally (because $\mathrm{dz}(0)=0)$.

Consider now the upper left component of inequality (26), which implies $A_{L}^{\top} P A_{L}-P<-2 \varepsilon I$, recall that (29) 
holds, and select

$$
\eta=\frac{\left|L^{\top} P L\right|+\left|L^{\top} P A_{L}\right|}{2 \varepsilon}
$$

to get

$$
\begin{aligned}
& \Delta V(e, \bar{\sigma})=e^{\top}\left(A_{L}^{\top} P A_{L}-P\right) e+q^{\top} L^{\top} P\left(2 A_{L} e+L q\right) \\
& +\zeta\left(\bar{\sigma}^{+}-\bar{\sigma}\right)+\eta \Delta V_{2}(e, \bar{\sigma}) \\
& \leq\left(-2 \varepsilon+\zeta\left|C^{\top} R C\right|\right)|e|^{2}-(1-\lambda) \zeta \bar{\sigma} \\
& +\underbrace{\left|L^{\top} P L\right||q|^{2}+2\left|L^{\top} P A_{L}\right||e||q|-2 \eta \varepsilon\left(|e|^{2}+|q|^{2}\right)}_{\leq 0} \\
& \leq-\varepsilon|e|^{2}-\zeta(1-\lambda) \bar{\sigma} \text {, if }(e, \bar{\sigma}) \notin \mathcal{M},\left(e^{+}, \bar{\sigma}^{+}\right) \notin \mathcal{M},
\end{aligned}
$$

thus completing the proof of Step (B).

Step $(C)$. To show that all solutions are bounded, we use the strong properties of function $V$ established in (30) and (32), in addition to bound (15) that clearly implies:

$$
\bar{c}_{1}\left(|e|^{2}+\bar{\sigma}\right) \leq V(e, \bar{\sigma}) \leq \bar{c}_{2}\left(|e|^{2}+\bar{\sigma}\right)
$$

for suitable positive scalars $\bar{c}_{1}, \bar{c}_{2}$. Then also using dynamics (22), we get:

$$
V\left(e^{+}, \bar{\sigma}^{+}\right) \leq \bar{c}_{2}\left(\left|e^{+}\right|^{2}+\bar{\sigma}^{+}\right) \leq M_{V} V(e, \bar{\sigma})
$$

where $M_{V}=\frac{\bar{c}_{2}}{\bar{c}_{1}} \max \left\{|A-L C|^{2}+|L|^{2}|C|^{2}+\left|C^{\top} R C\right|, 1\right\}$.

Consider now any solution starting in $\mathcal{M}$ and note that it remains in $\mathcal{M}$ due to forward invariance. Then equation (30) implies that the solution is bounded because $V$ is nonincreasing and also from the left bound in (33). If the solution starts outside $\mathcal{M}$, then $V$ is non-decreasing (from (32)) as long as the solution remains outside $\mathcal{M}$. If the solution jumps to $\mathcal{M}$, then (32) cannot be applied, but (34) guarantees a uniform bound on the possible growth of $V$ across the corresponding jump. After the solution enters $\mathcal{M}, V$ will never increase again, thereby establishing boundedness of the solution, as to be proven.

\subsection{Design}

In this section we propose two design procedures for optimizing the solution to (4c), (23), (24), and (35), thereby obtaining the observer parameters $L, W, R, \lambda$, based on convex optimization (SDP solvers). Such design paradigms can be easily extended to the continuoustime case of Section 2, and their use is illustrated in the simulation results of Section 4. The first design is called full stubborn design, wherein all the observer parameters, $\lambda, R, W$, and $L$ are simultaneously designed. The second design is seen as a stubborn augmentation of an existing (pre-selected) arbitrary observer gain $L$ ensuring that matrix $A-L C$ be Hurwitz. Such a stubborn design consists in selecting the remaining parameters $\lambda$, $R$, and $W$ of the observer augmentation.

In principle, any choice of $X, Y, \lambda \in[0,1), P=P^{\top}>0$, $R=R^{\top}>0, U>0$ diagonal, and $W>0$ diagonal such that the conditions (4c), (23), and (24) hold is a solution to the design problem for setting the adaptive observer (21) with gain (5). However, we note that all the constraints are homogeneous (they still hold if one scales all variables by a positive factor). Due to this fact, in lieu of $W>0$ it is preferable to set

$$
W>I
$$

in such a way to improve the numerical efficiency of the solver. For such reasons, this additional constraint does not restrict the feasibility set.

Full stubborn (FS) design. Since the matrix inequality (23) is an LMI with a fixed $\lambda$ and a large value of $\lambda$ is preferable as corresponding to a faster reaction to an increase of the output error, a generalized eigenvalue problem (GEVP for short, see [5]) in such a way as to minimize $\lambda$ subject to (4c), (23), (24), and (35) (note that in the continuous-time setting it consists in maximizing $\lambda>0$ ). The optimal $\lambda$ that results from the solution of such a relaxed problem has to be regarded as an upper bound, which can be used to restart the design procedure by fixing $\lambda$ to a fraction of such a bound. Thus, we can exploit the "degree of freedom" let by this relaxation by searching for the remaining unknowns in such a way to construct the stubborn observer in a convenient way.

Toward this end, various solutions can be put in practice after fixing $\lambda$. A design goal may be that of minimizing the norm of $R$ so that an input matched disturbance is less amplified by the observation law. To set the matrix $R$ as small as possible, one can minimize the scalar $\alpha>0$ under the additional constraint

$$
\left[\begin{array}{cc}
\alpha I & R^{\top} \\
R & \alpha I
\end{array}\right] \geq 0
$$

in such a way to get the minimum of $|R| \leq \alpha$. This formulation makes it possible to get a convenient tradeoff between decreasing $\lambda$ and suitably selecting the other parameters. As an alternative, one may minimize the $l_{2}$ gain from the disturbance $d$ to the estimation error $e$ [20]. Specifically, the problem reduces to minimizing $\gamma$ under the additional LMI condition

$$
\left[\begin{array}{cccc}
-P & 0 & C^{\top} & A^{\top} P-C^{\top} X^{\top} \\
\star & -\gamma I & D_{d}^{\top} & B_{d}^{\top} P-D_{d}^{\top} X^{\top} \\
\star & \star & -\gamma I & 0 \\
\star & \star & \star & -P
\end{array}\right]<0,
$$


where $\star$ denotes symmetric entries. Inequality (37) corresponds to the discrete-time version of the bounded real lemma, which ensures

$$
\left(e^{\top} P e\right)^{+}-\left(e^{\top} P e\right)+\frac{1}{\gamma}|y-\hat{y}|^{2}-\gamma|d|^{2} \leq 0 .
$$

It is worth noting that the $l_{2}$ design in (37) can be adopted also for the standard Luenberger observer, as it will be done in Section 4 for the selection of $L$ in the design of the stubborn augmentation observer.

Stubborn augmentation (SA) design. A feature that has been barely commented so far about the proposed stubborn design is that the proposed conditions (4c), (23), (24), and (35) preserve their convexity also when the gain $L$ has been fixed a priori to some generic selection. In particular, it is enough to replace $X$ by $P L$ in all its occurrences in (23), (24) and all the steps of our proofs remain valid. In particular, one can follow exactly the same design procedure of the previous FS construction and find the stubborn parameters $\lambda, R, W$ by first minimizing $\lambda$ and then also minimizing the norm of $R$ using constraint (36). The FS and SA setups will be discussed and illustrated in the next simulation section.

\section{Simulation results}

In the following, we will consider a passive tracking problem inspired by [9] with position measurements obtained by global positioning system (GPS) that may be subject to outliers. Specifically, the case study is based on the kinematic model of a mass point in two dimensions that moves on the plane and for which we have at disposal the measurements of the position along the axes, i.e., the discrete-time autonomous system with

$$
A=\left[\begin{array}{llll}
1 & T & 0 & 0 \\
0 & 1 & 0 & 0 \\
0 & 0 & 1 & T \\
0 & 0 & 0 & 1
\end{array}\right], \quad C=\left[\begin{array}{llll}
1 & 0 & 0 & 0 \\
0 & 0 & 1 & 0
\end{array}\right]
$$

where $T>0$ is the sampling period. Moreover, we suppose that the system is affected by four external disturbances. Specifically, the first and second disturbances act on the dynamics, whereas the third and fourth ones concern the position measurements:

$$
B_{d}=\left[\begin{array}{llll}
0 & 0 & 0 & 0 \\
1 & 0 & 0 & 0 \\
0 & 0 & 0 & 0 \\
0 & 1 & 0 & 0
\end{array}\right], \quad D_{d}=\left[\begin{array}{llll}
0 & 0 & 1 & 0 \\
0 & 0 & 0 & 1
\end{array}\right] .
$$

Here we comparatively show the performance obtained with a linear Luenberger observer (LLO), and compare it to the results obtained when performing the two designs suggested in Section 3.3, namely the FS and SA ones. Specifically, the SA observer is constructed on top of the gain chosen for the LLO. For the FS design, following the guidelines in Section 3.3, after finding the minimum $\lambda$ by solving a GEVP, we fix $\lambda$ as the square root of such a minimum value and we design an observer (21) by minimizing $|R|$ by way of $\alpha$ in (36). Such an observer will be denoted by FSO.

The LLO is constructed by minimizing the related $l_{2}$ gain that solves the convex problem in (37) with $P>0$, i.e., by minimizing the disturbance to output error gain $\gamma$ in (38). Finally, the SA design follows the guidelines at the end of Section 3.3 to design only parameters $\lambda, R, W$ of the stubborn estimator (21) having fixed a priori the gain $L$ to the value corresponding to the aforementioned $l_{2}$ optimal LLO selection. We will denote such an observer as $\mathrm{SAO}$.

We obtained the following design parameters for the FSO:

$$
\begin{aligned}
L_{\mathrm{FS}} & =\left[\begin{array}{cc}
1.9615 & 0 \\
0.5473 & 0 \\
0 & 1.9615 \\
0 & 0.5473
\end{array}\right], \quad W_{\mathrm{FS}}=\left[\begin{array}{ll}
1 & 0 \\
0 & 1
\end{array}\right], \\
R_{\mathrm{FS}} & =\left[\begin{array}{cc}
0.3124 & 0 \\
0 & 0.3124
\end{array}\right], \quad \lambda_{\mathrm{FS}}=0.4419,
\end{aligned}
$$

while for the LLO we obtained:

$$
L_{\mathrm{LL}}=\left[\begin{array}{cccc}
1.2496 & 0.4805 & 0 & 0 \\
0 & 0 & 1.2496 & 0.4805
\end{array}\right]^{\top},
$$

which has been used as a starting point to compute the following gains of the SAO:

$$
\begin{array}{rlrl}
L_{\mathrm{SA}} & =L_{\mathrm{LL}}, & W_{\mathrm{SA}}=\left[\begin{array}{ll}
1 & 0 \\
0 & 1
\end{array}\right], \\
R_{\mathrm{SA}}=\left[\begin{array}{cc}
0.3778 & 0 \\
0 & 0.3778
\end{array}\right], & \lambda_{\mathrm{SA}}=0.6250 .
\end{array}
$$

For the purpose of comparison, we will use boxplots, which are well-suited for illustrating the difference of performances among the considered estimators in quite an intuitive, "graphically-friendly" way. Specifically, we will show the boxplots of the root mean square error (RMSE) for each state variable with simulation runs of 

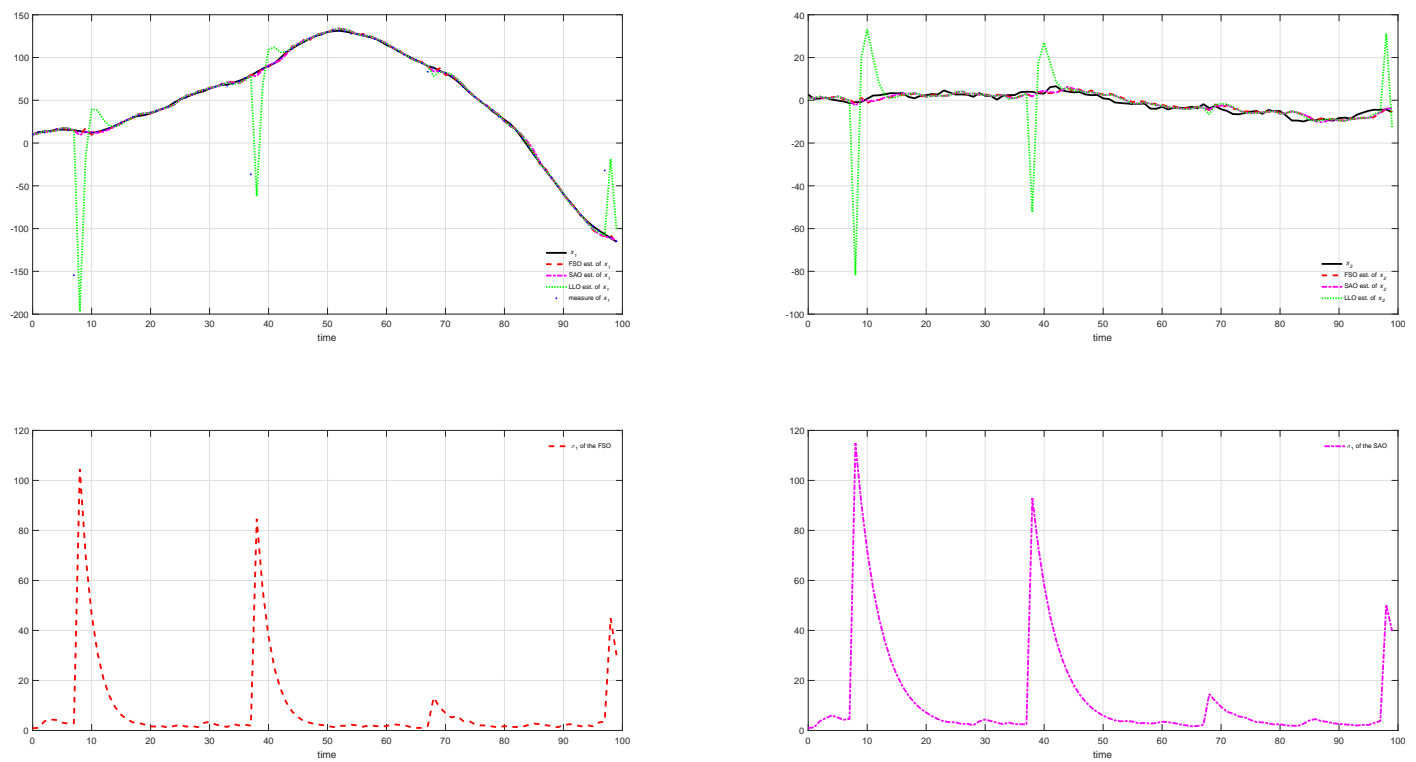

Fig. 2. Result of a simulation run with measurements corrupted by outliers.

Table 1

Medians of the RMSEs over 1000 runs for $x_{1}$ and $x_{2}$

\begin{tabular}{|c|c|c|c|c|c|c|c|c|c|c|}
\hline & \multicolumn{2}{|c|}{$\bar{T}=0, \bar{f}=0$} & \multicolumn{2}{|c|}{$\bar{T}=40, \bar{f}=10$} & \multicolumn{2}{|c|}{$\bar{T}=30, \bar{f}=100$} & \multicolumn{2}{|c|}{$\bar{T}=20, \bar{f}=10^{3}$} & \multicolumn{2}{|c|}{$\bar{T}=10, \bar{f}=10^{4}$} \\
\hline & $x_{1}$ & $x_{2}$ & $x_{1}$ & $x_{2}$ & $x_{1}$ & $x_{2}$ & $x_{1}$ & $x_{2}$ & $x_{1}$ & $x_{2}$ \\
\hline FSO & 1.9 & 1.7 & 2.1 & 1.7 & 2.3 & 1.7 & 2.5 & 1.8 & 106.8 & 34.2 \\
\hline $\mathrm{SAO}$ & 1.9 & 1.6 & 1.9 & 1.7 & 1.9 & 1.7 & 2.6 & 1.8 & 272.5 & 116.6 \\
\hline LLO & 1.6 & 1.6 & 2.0 & 1.7 & 15.2 & 6.7 & 186.5 & 79.9 & 2776.9 & 1186.8 \\
\hline
\end{tabular}
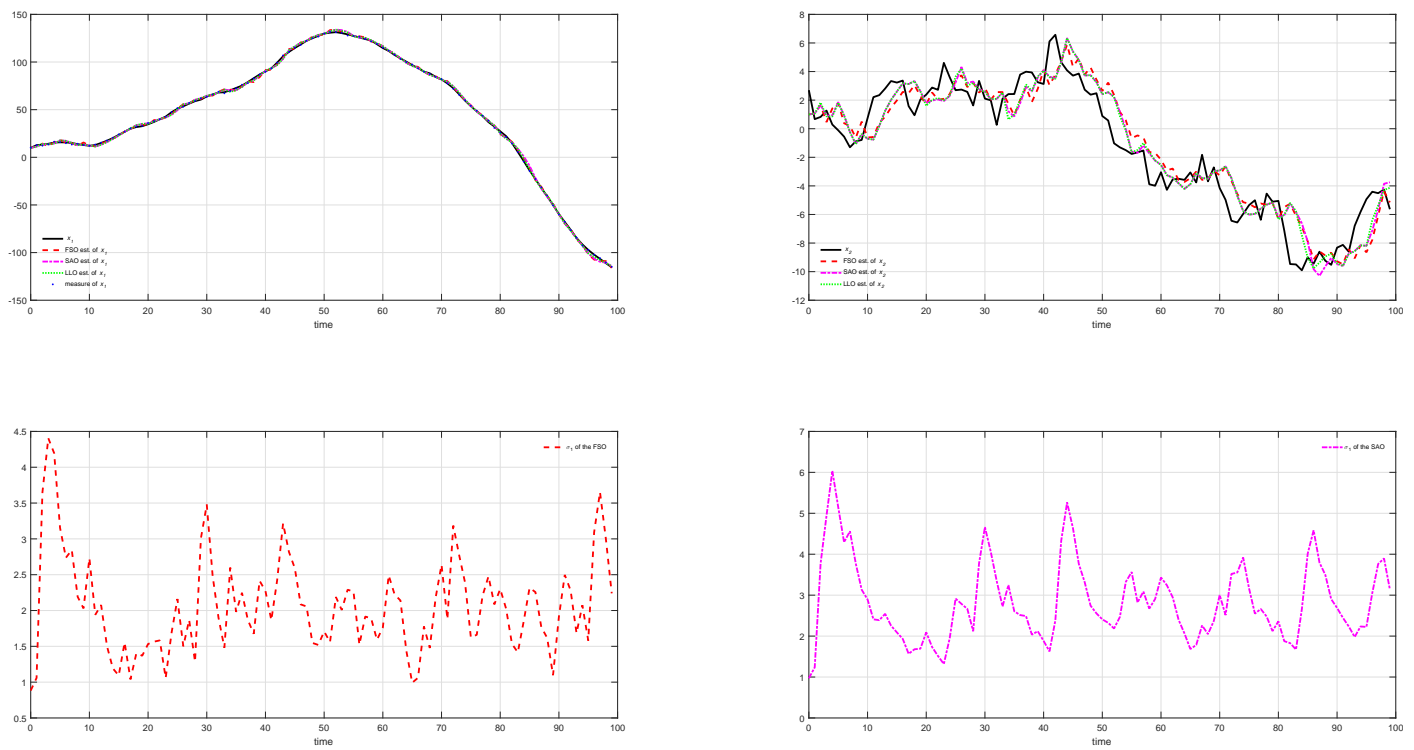

Fig. 4. Result of a simulation run with measurements without outliers.

length equal to $N$ time steps:

$$
\operatorname{RMSE}:=\left(\frac{1}{N} \sum_{t=0}^{N-1} e_{i, t}^{2}\right)^{1 / 2}
$$

where $e_{i, t}$ is the estimation error of the $i$-th state variable, $i=1,2,3,4$.

We consider a simulation setting with $T$ equal to $1 \mathrm{~s}$ and initial states randomly chosen around the initial esti- 

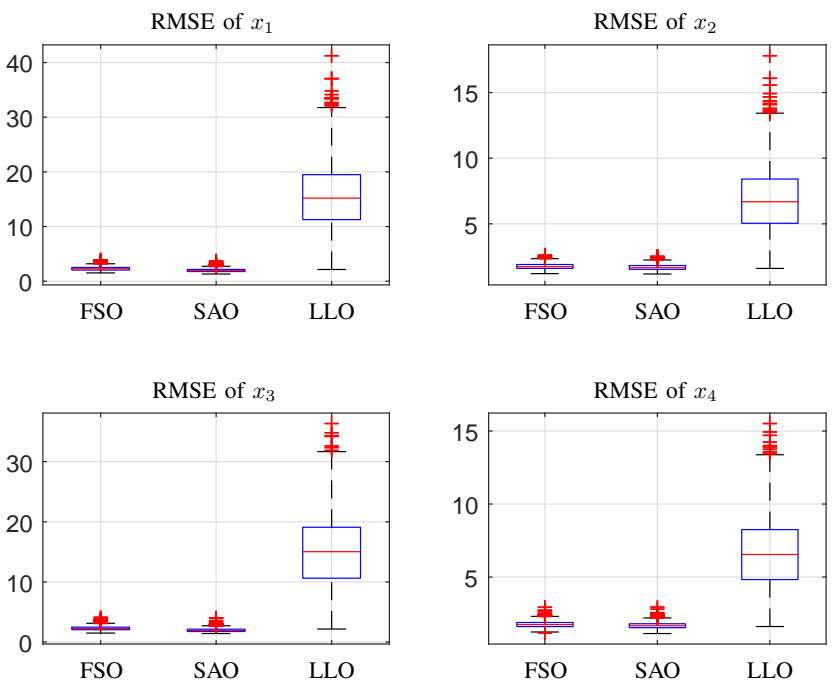

Fig. 1. Boxplots of the RMSEs over 1000 simulation runs with measurements corrupted by outliers $(\bar{T}=30$ and $\bar{f}=100)$.
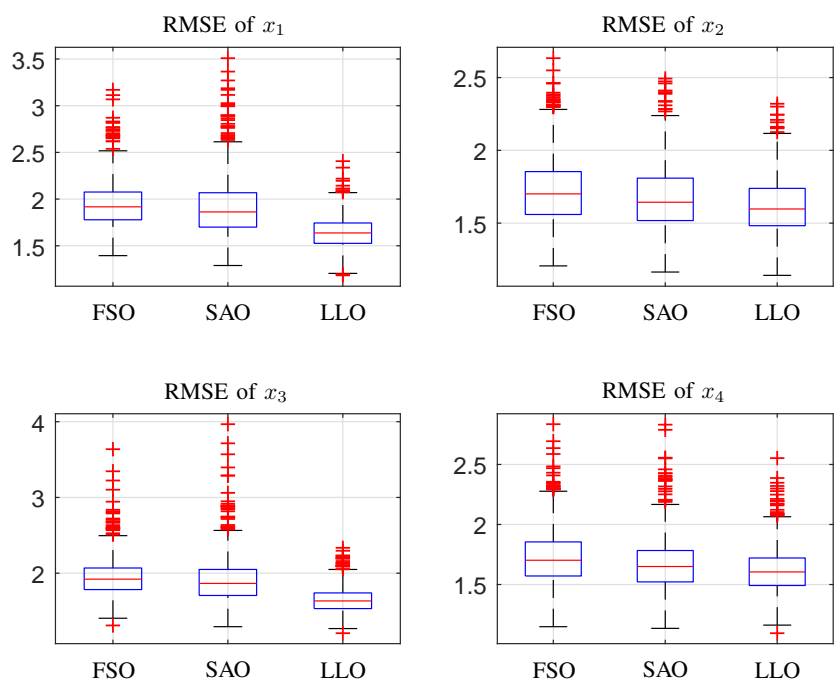

Fig. 3. Boxplots of the RMSEs over 1000 simulation runs with measurements without outliers.

mated state $(10,1,10,1)$ according to a normal distribution with covariance diag $(10,10,10,10)$. Zeromean, normal random noises with variance equal to 1 are chosen as systems noises. The measurement noises are modeled as zero-mean, normal random noises with variance equal to 0.5 , except in the case of outliers. The outlier occurrence is chosen to be periodic with a period equal to $\bar{T}$ and the time of the first outlier is randomly generated in the interval between 1 and $\bar{T}$. The outlier amplitudes are zero-mean, normal random noises with dispersion factor $\bar{f}$ as compared to the standard measurement noise, i.e., variance $0.5 \bar{f}^{2}$. Concerning such a simulation framework with $\bar{T}=30$ and $\bar{f}=100$, Figures 1 and 2 illustrate the distribution of the RMSEs over $L=1000$ simulation runs of length equal to $N=100$ and the time evolution of the three observers for a specific simulation run, respectively. Instead, Figures 3 and 4 show the same results in case of no outlier (we refer to such a setting with $\bar{T}=0$ and $\bar{f}=0$ in Table 1 ).

Table 1 shows the loss of performances in the medians of the RMSEs under different simulation regimes with an increase of outliers both in terms of frequency and amplitude. In the absence of outliers, the LLO performs better but the performances of FSO and SAO are quite close to that of the LLO. By contrast, an increase of outliers in terms of occurrence and magnitude causes a major failure of the LLO, whereas both the SAO and especially the FSO exhibit a much smoother performance degradation. Note that the SAO and FSO start showing significant differences only for large values of $\bar{f}$. The boxplots of Figures 1 and 3 show that on average the use of saturation in the FSO and SAO is extremely effective in making the state estimates almost insensitive to the measurement outliers, without sacrificing much the performances in the conditions of standard noise. Moreover, the proposed FSO design method based on the minimization of $|R|$ is more suitable than the pure $l_{2}$ criterion adopted for the LLO and the SAO, whenever one witnesses frequent measurement outliers. This fact can be explained by the higher rate of decrease $\lambda$ of the saturation threshold obtained from the FSO, as compared to that of the SAO, as shown by the time behaviors of $\sigma_{1}$ for the FSO and the SAO in Figure 2 (compare also the values reported in (39) and (40)).

As expected, in the absence of outliers, the LLO performs slightly better, but, in general, the saturation of the output error makes the estimates of the FSO and SAO much more robust to outliers. The proposed observers are well-suited for estimating the state variables in the presence of outliers, and, as compared with the Luenberger observer, the introduction of the saturation provides a meaningful performance improvement.

\section{Conclusions}

A novel approach to the design of a state estimator with saturation on the output injection has been proposed. Such an estimator has been called stubborn observer because of the intrinsic trend to decrease the saturation threshold in such way to make it robust to outliers in the measurements. The main contribution has concerned the proof of the global asymptotic stability of the estimation error dynamics in both the continuous and the discrete time cases. Moreover, we have provided convenient tools for the observer design by relying on an LMI-based framework. Simulations have confirmed the effectiveness of the stubborn observer in performing estimation with measurements affected by outliers. As a perspective of future work, we will investigate the input-to-state stability for the disturbance-to-error dynamics in the presence of measurement noise, as well as the extension of 
the proposed approach to estimate the state of nonlinear uncertain plants, possibly in a high-gain observers framework.

\section{References}

[1] A.D. Akkaya and M.L. Tiku. Robust estimation in multiple linear regression model with non-gaussian noise. Automatica, 44(2):407-417, 2008.

[2] A. Alessandri and M. Awawdeh. Moving-horizon estimation with guaranteed robustness for discrete-time linear systems and measurements subject to outliers. Automatica, 67:85-93, 2016.

[3] A. Alessandri and L. Zaccarian. Results on stubborn Luenberger observers for linear time-invariant plants. In European Control Conference, pages 2925-2930, Linz, Austria, July 2015.

[4] D. Astolfi, L. Marconi, and A.R. Teel. Low-power peakingfree high-gain observers for nonlinear systems. In European Control Conference, pages 1424-1429, Aalborg, Denmark, 2016.

[5] S. Boyd, L. El Ghaoui, E. Feron, and V. Balakrishnan. Linear Matrix Inequalities in System and Control Theory, volume 15 of Studies in Applied Mathematics. SIAM, Philadelphia, PA, 1994.

[6] M.S. Chong, D. Nesic, R. Postoyan, and L. Kuhlmann. Parameter and state estimation of nonlinear systems using a multi-observer under the supervisory framework. IEEE Trans. on Automatic Control, 60(9):2336-2349, 2015.

[7] F.H. Clarke. Optimization and Nonsmooth Analysis. Society for Industrial an Applied Mathematics, 1990.

[8] D. De Palma and G. Indiveri. Output outlier robust state estimation. Int. Journal of Adaptive Control and Signal Processing, 31(4):581-607, 2017.

[9] M.A. Gandhi and L. Mili. Robust Kalman filter based on a generalized maximum-likelihood-type estimator. IEEE Trans. on Signal Processing, 58(5):2509-2520, 2010.

[10] J.M. Gomes da Silva Jr and S. Tarbouriech. Anti-windup design with guaranteed regions of stability: an LMI-based approach. IEEE Trans. Aut. Cont., 50(1):106-111, 2005.

[11] J.P. Hespanha. Linear systems theory. Princeton university press, 2009.

[12] T. Hu and Z. Lin. Control systems with actuator saturation: analysis and design. Springer, 2001.

[13] T. Hu, Z. Lin, and B.M. Chen. An analysis and design method for linear systems subject to actuator saturation and disturbance. Automatica, 38(2):351-359, 2002.

[14] T. Hu, A.R. Teel, and L. Zaccarian. Anti-windup synthesis for linear control systems with input saturation: achieving regional, nonlinear performance. Automatica, 44(2):512-519, 2008.

[15] H.K. Khalil. Nonlinear Systems. Prentice Hall, USA, 3rd edition, 2002.

[16] F. Lauer, G. Bloch, and R. Vidal. A continuous optimization framework for hybrid system identification. Automatica, 47(3):608-613, 2011.

[17] H. Lei, J. Wei, and W. Li. A global observer for autonomous systems with bounded trajectories. Int. Journal of Robust and Nonlinear Control, 17(12):1088-1105, 2007.

[18] D. Luenberger. Observers for multivariable systems. IEEE Trans. on Automatic Control, 11(2):190-197, 1966.
[19] D.G. Luenberger. Observing the state of a linear system. IEEE Trans. on Military Electronics, 8(2):74-80, 1964.

[20] M. Massimetti, L. Zaccarian, T. Hu, and A.R. Teel. Linear discrete-time global and regional anti-windup: an LMI approach. Int. Journal of Control, 82(12):2179-2192, 2009.

[21] T. Passenbrunner, M. Sassano, and L. Zaccarian. Optimalitybased dynamic allocation with nonlinear first-order redundant actuators. Submitted to Automatica., 2015. See homepages.laas.fr/lzaccari/submitted/SassanoAuto15.pdf.

[22] D. Shi, T. Chen, and L. Shi. New Kalman filter and smoother consistency tests. Automatica, 49(10):3141-3144, 2013.

[23] E.D. Sontag. An algebraic approach to bounded controllability of linear systems. International Journal of Control, 39(1):181-188, 1984.

[24] S. Tarbouriech, G. Garcia, J.M. Gomes da Silva Jr., and I. Queinnec. Stability and stabilization of linear systems with saturating actuators. Springer-Verlag London Ltd., 2011.

[25] A.R. Teel. Further variants of the Astolfi/Marconi high-gain observer. In Proc. American Control Conference, pages 993998, Boston, USA, 2016.

[26] A.R. Teel and L. Praly. On assigning the derivative of a disturbance attenuation control Lyapunov function. Mathematics of Control, Signals, and Systems (MCSS), 13(2):95-124, 2000.

[27] W. Xu, E.-W. Bai, and M. Cho. System identification in the presence of outliers and random noises: A compressed sensing approach. Automatica, 50(11):2905-2911, 2014. 